\title{
The ILC Vertex Detector requirements
}

\author{
Auguste Besson* $\dagger$ \\ Université de Strasbourg, CNRS, IPHC UMR 7178, F-67000 Strasbourg, France \\ E-mail: abesson@in2p3.fr
}

\begin{abstract}
After few decades of R\&D, the International Linear Collider (ILC) project has reached a level of maturity proving the feasibility of the machine and of the detectors. The ILC physics goals cover a very wide and ambitious program including top-quark quark physics, electroweak precision measurements, direct and indirect searches beyond the Standard Model (BSM) like SUSY, dark matter manifestations, exotic particles and phenomena, etc., and an extensive Higgs physics program covering mass, couplings to fermions and bosons, quantum numbers and total width measurements. These measurements are expected to reach an unprecedented level of precision, which will allow probing physics BSM, since typical deviations from the Standard Model are expected to be within the ILC sensitivity. To accomplish the ambitious physics program of the ILC, the vertex detector will be essential for providing the necessary physics performances in terms of flavour tagging, displaced vertex charge determination and low momentum tracking capabilities. Taking advantage of the much less demanding running conditions at the ILC than at hadron colliders like LHC, the vertex detector is expected to reach particularly high performances as far as spatial resolution and material budget are concerned (typical impact parameter resolution of the order of 5 microns and material budget in the order of $0.15-0.2 \%$ of radiation length per layer). In addition, the particular time structure of the beams has major consequences on the specifications of the detectors and their read-out architecture. Finally, the beam related background of the ILC, which translates into a high rate of low momentum- $e^{-} e^{+}$pairs hitting the vertex detector, drives the expected occupancy (and the related necessary read-out speed) as well as the radiation load. This article focuses on the vertex detector requirements driven from the stringent physics and experimental constraints of the ILC. Wherever different, the aspects to each detector concepts (SiD and ILD) are discussed.
\end{abstract}

The 25th International workshop on vertex detectors

September 26-30, 2016

La Biodola, Isola dâĂŹElba, ITALY

${ }^{*}$ Speaker.
${ }^{\dagger}$ On behalf of $\mathrm{SiD}$ and ILD vertex detector $\mathrm{R} \& \mathrm{D}$ groups

(c) Copyright owned by the author(s) under the terms of the Creative Commons 


\section{The International Linear Collider (ILC)}

\subsection{ILC context}

This article provides an overview of the vertexing detector requirements at the International Linear Collider (ILC). After few decades of R\&D, the ILC project has reached a level of maturity proving the feasibility of the machine and the detectors construction. In the last 7 years, several significant milestones have been reached. In 2009, two detector projects (ILD \& SiD) provided a Letter Of Intent (LOI)[1] summarizing the detector concepts, while in 2012, the accelerator Technical Design Report (TDR) and the Detailed Baseline Design (DBD) were published [2]. The ILC is currently at a turning point of its history. A negotiation process is expecting to be pursued in the coming years and could lead to the beginning of the construction work around 2020 and to first collisions at the beginning of the 2030 decade.

The ILC physics goals cover a wide and ambitious program including top quark physics, electroweak precision measurements, direct and indirect searches beyond the Standard Model (BSM) like SUSY, dark matter searches, exotic searches, etc., and a complete and extensive Higgs physics program covering mass, couplings to fermions and bosons, quantum numbers and total width measurements. The expected level of precision for the majority of them will reach the percent level and will allow probing physics BSM in a model independent way, since typical deviations are expected to be in this range [3].

As an $e^{+} e^{-}$collider, the ILC benefits from a very clean environment, with almost no QCD background, a well known initial state and fully reconstructible channels. In addition the ILC will be able to lean on precise theoretical calculations at the per mil level [4] [5] [6]. The machine will allow a tunable center of mass energy and will provide polarized beams offering a large physics program from $\sqrt{s}=250,350$ up to $500 / 550 \mathrm{GeV}$. A $\sqrt{s}=1 \mathrm{TeV}$ upgrade is also possible. This will add a lot of flexibility. For instance, a scan of the top pair production threshold can also be performed to measure the top quark properties.

At last but not least, the cross-sections of primary interest are generally only one to two order of magnitude below the total cross-section (e.g. at $\sqrt{s}=500 \mathrm{GeV}, \sigma\left(e^{+} e^{-} \rightarrow Z H\right) \simeq 100 \mathrm{fb}$ while $\left.\sigma\left(e^{+} e^{-} \rightarrow q \bar{q}\right) \simeq 10^{4} \mathrm{fb}\right)$. This is much more favorable compared to the 10 orders of magnitude of the same ratios at LHC. The low cross-sections will therefore be compensated by favorable Signalto-Noise ratios. However, most of the measurements will be limited by statistical precision which implies that for a given analysis even fully hadronic decay final states will have to be included.

\subsection{ILC experimental conditions}

The linear character of the ILC governs the beam structure which will be composed of 5 trains per second, of $\simeq 1300 / 2600$ bunches (depending on the options), each bunch being separated by approximately 330/550 ns (depending on the options). Between each train, there is a beamless time of around $200 \mathrm{~ms}$. This particular beam structure has major consequences on the specifications of the detectors and their read-out. It allows a complete triggerless detector, postponing the read-out between the trains and a decrease of the average power consumption by switching off (at least partially) the detectors between trains (the so called power pulsing).

The other major experimental feature is the background induced by the beam interaction with the electromagnetic field of the other beam. This background, so called beamstrahlung, is respon- 
sible of photon emission which finally translates into massive $e^{+} e^{-}$pair creations. Those electrons which hit the vertex detector can come from either the interaction region (80\% of the case) or the forward region (backscattered particles). They can also come from the detector region itself (including the beam pipe). The momentum of the electrons hitting the vertex detector typically lies in the $10-100 \mathrm{MeV} / \mathrm{c}$ range. The beamstrahlung is also responsible for a random center of mass energy loss (typically $1 \%$ in average at $\sqrt{s}=250 \mathrm{GeV}$ ). It finally drives completely the occupancy and determines directly the expected radiation level in the inner detectors:

- Radiations: Though moderate compared to the LHC, the expected radiation level remains significant. Among all the detectors of the central region, the first layer of the vertex detector will receive the highest radiations. The ionizing radiation are expected to be of the order of $100 \mathrm{kRad} / \mathrm{year}$ whereas the non ionizing radiations are expected to be of the order of $10^{11} n_{e q}(1 \mathrm{MeV}) / \mathrm{cm}^{2} /$ year, including safety factors.

- Occupancy: The tracks coming from physics events are completely negligible in regards to the occupancy induced by the background. This occupancy, which depends on the beam structure options and on $\sqrt{s}$, is also very sensitive to the geometry layout of the interaction region. Several studies have been performed to estimate the occupancy on the different layers of the vertex detector [2][7]. A typical value obtained is $5 \mathrm{hits} / \mathrm{cm}^{2} / \mathrm{Bunch}$ crossing on the first layer. However, since there is no experimental data (with the expected beam structure) to validate it, the simulations suffers from large uncertainties. In addition the final rates depends highly on the beam structure and the interaction region design which is not completly finalized. For example, modifications in the interaction region geometry layout can typically change the expected background up to a factor 2 [8]. As a consequence, applying a safety factor (at least $\times 5$ ) on these estimates is considered very necessary by the community. This is a typical safety factor applied in previous experiments [9].

\subsection{Vertex detector requirements}

The general philosophy of both detectors (SiD and ILD) is based on a Particle Flow Algorithm (PFA) approach. PFA is not a new idea and is already used extensively in LHC experiments but it is planned to be developed to an unprecedented level. This will demand unprecedented precision detectors, with a highly granular calorimeter, excellent momentum resolution and vertexing capabilities. Compared to LHC detectors, performances will have to be significantly enhanced on the jet energy resolution (typically by a factor $\simeq 3$ with respect to LHC), the momentum resolution (factor $\simeq 10$ with respect to LHC) and the vertex reconstruction (with a pixel size and a material budget reduced by a factor $\simeq 10$ with respect to LHC). At last but not least, both detectors will be able to allow the push pull operation since only one interaction point is foreseen.

To fulfill the ambitious physics program, the vertex detector will play a crucial role. The figure of merit of the vertex detector which is the impact parameter resolution is expected to be:

$$
\sigma_{i p}=5 \mu m \oplus \frac{10}{p \beta \sin \theta^{3 / 2}} \mu m \cdot G e V / c
$$

The vertex detector should therefore sustain the following capabilities: 
- a spatial resolution of the inner layer close to $\simeq 3 \mu \mathrm{m}$ (which corresponds to a pitch $\simeq 17 \mu \mathrm{m}$ ),

- a low material budget to minimize multiple scattering, typically $\simeq 0.15 \% X_{0} /$ layer,

- a low momentum tracking capabilities (even for $p_{T}$ below $100 \mathrm{MeV} / \mathrm{c}$ ),

- a good angular coverage,

- an enhanced heavy flavour tagging (b-jets,c-jets, $\tau$ ) with a very high efficiency and purity,

- a moderate power consumption $(\langle P\rangle \simeq 12 \mathrm{~W}$ accounting for a $3 \%$ duty cycle) for the complete vertex detector,

- a detector able to comply with radiation hardness as ionizing radiations are expected to be of the order of $100 \mathrm{kRad} / \mathrm{year}$ and fluence is expected to be of the order of $10^{11} n_{e q}(1 \mathrm{MeV}) /$ year, on the first layer, taking into account a safety factor.

- a detector able to sustain possible pick up noise and single event effects.

- a time resolution and data rate output defined by the running conditions and the read-out strategy.

- an alignment at the micron level capabilities.

Putting all these specifications together represents a very difficult challenge since no technology fulfill easily the requirements all together. This makes exploring all the different technology options and a long term R\&D program mandatory. However, the running conditions (radiation hardness and occupancy) are much less demanding than those at LHC which favors technologies able to sustain high resolution and very low material budget.

\section{The SiD and ILD detectors}

The SiD and ILD share some common features. They are both designed to exploit fully Particle Flow Algorithms (PFA). The electromagnetic and the hadronic calorimeters are designed to be placed inside the coil to optimize track-calorimeter cluster associations in the PFA approach. They are also both expected to be equipped with forward trackers, muon detectors an high precision low mass vertex detectors. The beam pipe is expected to be made of $\simeq 500 \mu \mathrm{m}$ of Beryllium in the interaction region. On the other hand, they also contain substantial differences in their design. More details can be found in [2].

\section{1 $\mathrm{SiD}$ tracking and vertexing system}

The SiD has a relatively compact radius compared to ILD. SiD includes a 5 Tesla magnetic field solenoide (in order to optimize vertex performances and compensate the smaller size). Its vertex detector is designed with a short barrel and endcap disks. The tracking detector is based on a silicon only (single sided microstrips) approach with few high precision points to enhance robustness, minimize risks and allow time stamping. The relative small size is also expected to be more cost efficient. This approach leads to a tracker composed of 5 layers in the barrel and 5 disks 


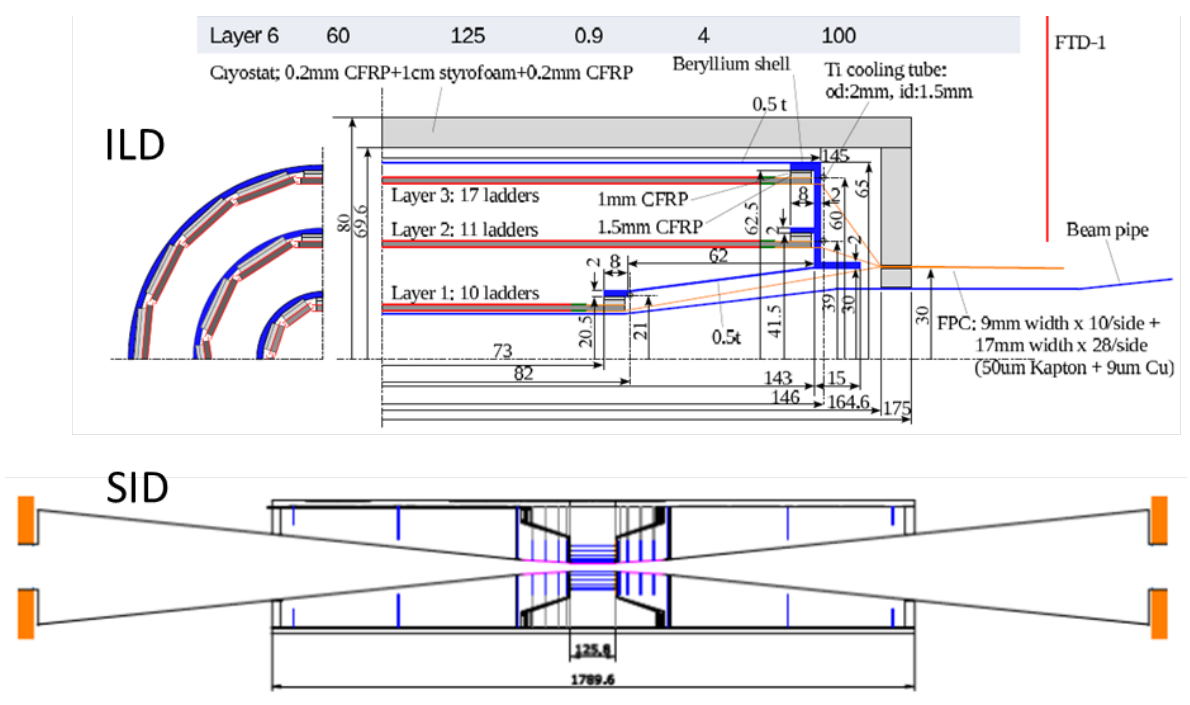

Figure 1: ILD and SiD schemes of the vertex detector

(conical and annular) in the forward regions. One challenge consists in minimizing the material budget with a low mass support.

The vertex detector itself will be made of 5 short barrels of silicon pixels (with a radius from $R=14 \mathrm{~mm}$ to $R=60 \mathrm{~mm}$ and with a length of $|Z|=63 \mathrm{~mm}$ ). The barrel will be complemented by 4 disks at short distance and 3 disks further (cf. fig.1).

\subsection{ILD tracking and vertexing system}

The ILD is designed with a long barrel and endcap disks surrounded by a 3.5 Tesla magnetic field. The main tracker consist of a TPC in order to optimize particle separation, pattern recognition (thanks to a large number of hits) and to allow $d E / d x$ measurement capabilities. The TPC is surrounded by intermediate silicon detectors in the central (SIT, SET) and the forward (ETD, FTD) regions in order to improve the tracking resolution, the calibration and the alignment. In addition, it makes the linking between the tracks and the calorimeter clusters much easier. Furthermore, it will allow the time stamping of the tracks. Besides the resolution and the read-out, the challenges of the $\mathrm{R} \& \mathrm{D}$ consist in maintaining the material budget small, minimize the power consumption and building a push pull compatible tracking system.

The long barrel approach guides also the vertex detector design. The main option consists in 3 double sided ladders (radius from $R=16 \mathrm{~mm}$ to $R=60 \mathrm{~mm}$ ) in order to optimize the material budget with respect to self alignment and stand alone tracking capabilities. The inner ladder will be shorter $(|Z|=62.5 \mathrm{~mm})$ but the two outer ladders will cover a larger polar angle $(|Z|=125 \mathrm{~mm})$. Another option, with 5 single sided layers in order to minimize the material budget is also being considered.

\section{The ILC vertex detector challenges}

The challenge of the ILC vertex detector can be summarized with a question: how to design a detector able to sustain the expected occupancy while maintaining the performances in terms 
of granularity (spatial resolution of the inner layer $\sigma_{s p} \simeq 3 \mu \mathrm{m}$ ) and material budget (radiation length $X_{0} \simeq 0.15 \% X_{0} /$ layer) without compromising the power consumption requirements $(<P>\simeq$ $12 W$ with possibly power pulsing) ? There are mainly three possible answers to this question which are related to the different read-out strategies.

\subsection{Read-out strategies}

Taking advantage of the beam structure, the vertex detector could be read out only during the long beamless time $(199 \mathrm{~ms}$ ) between each train. Then, one read out would integrate a complete train. The relaxed constrain on the read out speed allows to foresee a very granular detector. The improved resolution should compensate, at least partially, the lack of bunch tagging and the high occupancy with better hit separation. At last but not least, small granularity could allow a possible tagging of the beam background tracks. Those are usually low momentum tracks and have a larger incident angle in the local frame of the detector. Therefore, the charge deposition of those tracks is usually elongated in one dimension of the detector plane. The modified cluster shape properties could be used to tag and/or reject those hits.

The second approach consists in disentangling the time stamping and the read out speed by storing hits with memories inside the pixels. In principle, single bunch tagging could be reached although it may enter into conflict with the granularity of the sensors. The SiD follows this approach.

The last approach tries to find a compromise between spatial and time measurement resolution by reading the sensors as fast as possible in a continuous mode during the train. Power consumption is generally increased, depending on the technology and the read-out mode. As a consequence, power pulsing might be mandatory with this read out strategy, although a prority encoding read out approach could allow a moderate power consumption [12].

Other options are considered like combinations of different technologies and/or read out strategies to find the optimum in the spatial vs time resolution parameter space.

The tab.1 summarizes the three main possible read-out strategies. One should keep in mind that the respective performances are just given as rough indications. Significant changes may occur thanks to the evolving technologies.

\begin{tabular}{|c|c|c|c|c|c|}
\hline Strategy & Power & Time resolution & Spatial resolution & Advantages & Caveats \\
\hline \multicolumn{6}{|c|}{ Fine pixels (e.g. FPCCD) } \\
\hline $\begin{array}{c}\text { Read-out } \\
\text { between train }\end{array}$ & low & 1 complete train & $\begin{array}{c}\lesssim 1 \mu m \\
(\simeq 5 \mu \mathrm{m} \text { pitch })\end{array}$ & $\begin{array}{c}\text { Spatial resolution } \\
\text { Hit separation } \\
\text { Beam background tagging } \\
\text { (cluster shape) }\end{array}$ & $\begin{array}{c}\text { No time stamping } \\
\text { Possible high occupancy } \\
\times 16 \text { more pixels }\end{array}$ \\
\hline \multicolumn{6}{|c|}{ In pixel time stamping (e.g. chronopixels, SOI) } \\
\hline $\begin{array}{c}\text { Read-out } \\
\text { between train }\end{array}$ & low & $\begin{array}{l}\text { single or few } \\
\text { bunches } \\
(\gtrsim 0.5 \mu s)\end{array}$ & $\begin{array}{c}\gtrsim 5 \mu \mathrm{m} \\
(\simeq 20-25 \mu \mathrm{m} \text { pitch })\end{array}$ & $\begin{array}{c}\text { Hit time stamping } \\
\text { Well suited to outer layers }\end{array}$ & $\begin{array}{c}\text { Time stamping storage } \\
\text { in conflict } \\
\text { with granularity }\end{array}$ \\
\hline \multicolumn{6}{|c|}{ Continuous read-out during train with rolling shutter or priority encoding. (e.g. DEPFET, CMOS) } \\
\hline $\begin{array}{l}\text { Read-out } \\
\text { during train }\end{array}$ & high & $\begin{array}{c}\text { few to } \\
10^{s} \text { bunches } \\
(5-50 \mu s)\end{array}$ & $\begin{array}{c}\lesssim 3 \mu m \\
(\simeq 17 \mu m \text { pitch })\end{array}$ & $\begin{array}{l}\text { Time and Spatial } \\
\text { resolution } \\
\text { compromise }\end{array}$ & $\begin{array}{c}\text { Power cycling } \\
\text { probably } \\
\text { needed }\end{array}$ \\
\hline
\end{tabular}

Table 1: Summary of the different read-out strategies for the ILC vertex detector 


\subsection{Technology R \& D}

At the present time, many technology options are still being considered. Already several mature technologies are approaching the specifications and some of them are already used in real experiments ([10],[11],[12]). Those experiments offer less demanding requirements but are in the same order of magnitude as the one of the ILC. More details on the technologies can be found in [13] (presented in this conference) and in [14][15][16][17][18], [19]. The $R \& D$ will continue to assess the detector performances and to reinforce the robustness with respect to beam background. At last but not least, all possible integrations issues will be studied carefully.

\subsection{Integration challenges}

The total material budget remains a challenge. Today, $50 \mu \mathrm{m}$ thick sensors are routinely produced (CMOS, DEPFET, etc.). In addition, already fully functional ladders have been fabricated (or even used in experiments) reaching the $0.2-0.3 \% X_{0}$ /layer range in the sensitive acceptance. It is believed that no show stopper will occur on this issue.

The cooling system will depend on the technology choice. Besides, the baseline consist in air cooling which is expected to be able to extract the total power dissipation of the vertex detector (few tens of watts). More specific developments are being pursued (e.g. micro-channel cooling for DEPFET, 2 phase- $\mathrm{CO}_{2}$ cooling system for Fine Pixel CCDs, etc.). Finally, the power pulsing, which consists in switching off (at least partially) the detector during the beamless time in order to reduce the power consumption, is also studied and may be mandatory for several technologies.

At last but not least, the alignment procedure will be deeply studied since the desired alignment precision has to reach an unprecedented level, of $O(\mu \mathrm{m})$. The push pull of the detector will make this procedure even more challenging.

\section{Conclusion}

On the present day, the ILC is a mature project. The DBD document [2] demonstrated the feasibility of the detectors. The ILD and the SiD developed two very complementary approaches. However, $R \& D$ on the vertex detectors (among others) is still ongoing. Many options are considered as viable as far as technologies, geometries and read out strategies are concerned. The coming years will be devoted to refine even more precisely the requirements putting in balance the detector performances, the robustness with respect to the beam background, the reliability, the integration considerations and the costs. Optimizations will rely on complete physics studies and optimized algorithms.

The author and the ILC vertex $R \& D$ groups thank very warmly the conference organizers for giving them the opportunity to present their work. The author thanks also M. Winter, M. Vos, A. Ishikawa and J. Goldstein for useful discussions.

\section{References}

[1] SiD Letter of Intent. The SiD concept group, (2010). http://arxiv.org/abs/0911.0006 International Large Detector Letter Of Intent. The ILD concept group, (2010). http://arxiv.org/abs/1006.3396 
[2] ILC Technical Design Report, (2013). http://www.linearcollider.org/ILC/Publications/Technical-Design-Report

[3] Comparison of LHC and ILC Capabilities for Higgs Boson Coupling Measurements, M.E. Peskin, arXiv:1207.2516 [hep-ph] SLAC-PUB-15178 (2012).

[4] Physics Case for the International Linear Collider, ILC-NOTE-2015-067, LCC Physics Working Group (2015).

[5] The Anatomy of Electro-Weak Symmetry Breaking. I: The Higgs boson in the Standard Model, Abdelhak Djouadi, arXiv:hep-ph/0503172 and arXiv:hep-ph/0503173 (2005).

[6] F.M. Renard, Basics of electron-positron collisions, Ed. Frontières, Gif-sur-Yvette, France, 1981; P.M. Zerwas, Physics with an e+e- Linear Collider at High Luminosity in: Particle Physics: Ideas and Recent Developments, J.J. Aubert et al. (eds.), Kluwer Academic Publishers, Amsterdam, (1999).

[7] Beam-Induced Backgrounds in Detectors at the ILC, PhD thesis, A.Vogel (2008). http://www-library.desy.de/preparch/desy/thesis/desy-thesis-08-036.pdf

[8] Vertex Detector Occupancy Studies, B. Schumm and C Milke, LCWS2015, https://lcws15.triumf.ca/ (2015).

[9] Technical Design Report for the Upgrade of the ALICE Inner Tracking System, The ALICE Collaboration, J. Phys. G: Nucl. Part. Phys. 41087002 (2014) page 9.

[10] Benjamin Schwenker, Development and construction of the Belle II DEPFET pixel detector, this conference.

[11] L. C. Greiner, STAR MAPS Vertex Detector operational experience, this conference.

[12] Stefania Beole, The upgrade of the ALICE ITS, this conference.

[13] J. Goldstein, Development of detector technologies for ILC vertexing, this conference.

[14] DEPFET active pixel detectors for a future linear e+e- collider, The DEPFET collaboration, (2013) http://arxiv.org/abs/1212.2160

[15] Development of CMOS pixel sensors for tracking and vertexing in high energy physics experiments, S. Senyukov et al. http://arxiv.org/abs/arXiv:1402.2172

MISTRAL \& ASTRAL : Two CMOS Pixel Sensor Architectures Dedicated to the Inner Tracking System of the ALICE Experiment, F. Morel et al., JINST 9 (2014) C01026.

A reticle size CMOS pixel sensor dedicated to the STAR HFT, I. Valin et al., JINST 7 (2012) C01102

[16] Fine Pixel CCD Option for the ILC Vertex Detector, Y. Sugimoto, International Linear Collider Workshop - Stanford, U.S.A, (2005)

Test of fine pixel CCDs for ILC vertex detector, Y. Sugimoto et al., KEK/J-PARC-PAC 2013-2, (2013).

[17] Chronopixel Vertex Detectors for Future Linear Collider, C. Baltay et al., (2011). http://arxiv.org/abs/1109.2811

[18] Y. Arai et al., In the Proceedings of International Symposium on Detector Development for Particle, Astroparticle and Synchrotron Radiation Experiments (SNIC 2006), Menlo Park, California, 3-6 Apr 2006, pp 0016.

[19] V. Re, Vertical integration technologies for tracking detectors, this conference. 